PROCEEDINGS OF THE

AMERICAN MATHEMATICAL SOCIETY

Volume 103, Number 3, July 1988

\title{
THE SPACE OF CROSS SECTIONS OF A BUNDLE KATSURO SAKAI
}

(Communicated by Doug W. Curtis)

\begin{abstract}
Let $B$ be a nondiscrete compactum, $Y$ a separable complete metrizable ANR with no isolated point and $p: X \rightarrow B$ a locally trivial bundle with fiber $Y$ admitting a section. It is proved that the space $\Gamma(X)$ of all cross sections of $p: X \rightarrow B$ is an $l_{2}$-manifold.
\end{abstract}

0. Introduction. Through the paper, spaces are separable metrizable and maps are continuous. Let $p: X \rightarrow B$ be a locally trivial bundle with fiber $Y$, that is, each point $b \in B$ has a neighborhood $U$ and a homeomorphism $\varphi: U \times Y \rightarrow p^{-1}(U)$ such that $p \varphi=\pi_{U}$, the projection to $U$. A map $s: B \rightarrow X$ is called a cross section of $p: X \rightarrow B$ provided $p s=$ id. The space of all cross sections of $p: X \rightarrow B$ with compact-open topology is denoted by $\Gamma(X)$. Then $\Gamma(X)$ is a closed subspace of the space $C(B, X)$ of all maps from $B$ into $X$. If $B$ is compact and $d$ is a compatible metric for $X$, the topology of $\Gamma(X)$ (and $C(B, X)$ ) is induced by the sup-metric

$$
\hat{d}(f, g)=\sup \{d(f(b), g(b)) \mid b \in B\} .
$$

A manifold modeled on Hilbert space $l_{2}$ is called an $l_{2}$-manifold. In this note, we prove the following

MAIN THEOREM. Let $B$ be a nondiscrete compactum, $Y$ a complete metrizable ANR with no isolated point and $p: X \rightarrow B$ a locally trivial bundle with fiber $Y$ admitting a section. Then $\Gamma(X)$ is an $l_{2}$-manifold.

For the trivial bundle $\pi_{B}: B \times Y \rightarrow B$, the space $\Gamma(B \times Y)$ can be regarded as the space $C(B, Y)$. Thus the space $C(B, Y)$ is an $l_{2}$-manifold if $B$ is a nondiscrete compactum and $Y$ is a complete-metrizable ANR with no isolated point. This is a generalization of Eells-Geoghegan-Toruńczyk's result $\left[\mathbf{E}, \mathbf{G e}, \mathbf{T o}_{\mathbf{1}}\right]$.

The author would like to thank Doug Curtis for helpful comments.

1. Preliminaries. Our proof is based on the following:

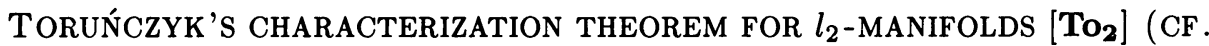
$\left.\left[\mathrm{To}_{3}\right]\right)$. A complete-metrizable ANR $X$ is an $l_{2}$-manifold if and only if $X$ has the discrete approximation property, that is, for each map $f: \bigoplus_{n \in \mathbf{N}} I^{n} \rightarrow X$ of the free union of $n$-cells $(n \in \mathbf{N})$ into $X$ and each map $\varepsilon: X \rightarrow(0,1)$ there is a

Received by the editors February 27, 1987 and, in revised form, April 22, 1987. Presented to the Mathematical Society of Japan, April 2, 1988.

1980 Mathematics Subject Classification. Primary 58D15, 57N20, 55F10.

Key words and phrases. Space of cross sections, locally trivial bundle, ANR, $l_{2}$-manifold.

This work was done while the author was visiting the University of California at Santa Barbara during July 1986-March 1987. 
map g: $\bigoplus_{n \in \mathbf{N}} I^{n} \rightarrow X$ with $d(f(x), g(x))<\varepsilon f(x)$ for all $x \in \bigoplus_{n \in \mathbf{N}} I^{n}$ and $\left\{g\left(I^{n}\right) \mid n \in \mathbf{N}\right\}$ a discrete family in $X$.

In order to verify the discrete approximation property of $\Gamma(X)$, we use the following easy modification of [DT, Remark 2] (cf. Proof of [DT, Lemma 1]).

LEMMA 1. Let $X=(X, d)$ be a locally path-connected metric space with a tower $X_{1} \subset X_{2} \subset \cdots(\subset X)$ satisfying the following properties:

(a) Given a compactum $A \subset I^{n}$, a map $f: I^{n} \rightarrow X$ with $f(A) \subset X_{i}$ and $\varepsilon>0$, there is a map $g: I^{n} \rightarrow X_{j}$ into some $X_{j} \supset X_{i}$ with $f|A=g| A$ and $\hat{d}(f, g)<\varepsilon$;

(b) Given $\varepsilon>0$, there is a $\delta>0$ such that any map $f: I^{n} \rightarrow X_{i}$ is $\varepsilon$-homotopic to a map $g: I^{n} \rightarrow X_{j}$ in some $X_{j} \supset X_{i}$ with $\operatorname{dist}\left(f\left(I^{n}\right), g\left(I^{n}\right)\right)>\delta$.

Then $X$ has the discrete approximation property.

By the next lemma, we can treat noncompact ANR like a compact one.

LEMMA 2 [Mi, LEMMA 2.1]. Every metric ANR $Y=\left(Y, d_{0}\right)$ has a compatible metric $d \geq d_{0}$ with the following property:

(h) For each $\varepsilon>0$, there is a $\delta=\delta(\varepsilon)>0$ such that any two $\delta$-near maps of an arbitrary space to $Y=(Y, d)$ are $\varepsilon$-homotopic

Let $p: X \rightarrow B$ and $q: Z \rightarrow B$ be given maps. A map $f: Z \rightarrow X$ is said to be fiber-preserving (f.p.) if $p f=q$. We call $p: X \rightarrow B$ an absolute neighborhood fiber extensor (ANFE) if any f.p. map $f: A \rightarrow X$ of a closed set in an arbitrary space $Z$ with a map $q: Z \rightarrow B$ can be extended to an f.p. map $\tilde{f}: U \rightarrow X$ of a neighborhood of $A$ in $Z$. A map $p: X \rightarrow B$ is called an absolute neighborhood fiber retract (ANFR) provided whenever $X$ is embedded in a space $Z$ with a map $q: Z \rightarrow B$ as a closed set with $p=q \mid X$ there is an f.p. retraction $r: U \rightarrow X$ of a neighborhood of $X$ in $Z$ onto $X$. A map $p: X \rightarrow B$ is an ANFR if and only if $p$ is an ANFE. (See $\S 1$ of [Ya].) By [Ya, 1.2], we have the following

LEMMA 3. Any locally trivial bundle $p: X \rightarrow B$ with $A N R$ fiber is an ANFR, that is, an $A N F E$.

We refer to [Du, Chapter XII] for function spaces and to [Hu] for ANR's.

2. Proof of main theorem. First we show that $\Gamma(X)$ is an ANR. Let $f: A \rightarrow$ $\Gamma(X)$ be a map from a closed set in a space $Z$. Then $f$ induces the map $F: A \times B \rightarrow$ $X$ with $p F=\pi_{B}$, the projection. By Lemma $3, F$ extends to a map $\widetilde{F}: W \rightarrow X$ from a neighborhood of $A \times B$ in $Z \times B$ with $p \widetilde{F}=\pi_{B}$. From compactness of $B$, there is a neighborhood $U$ of $A$ in $Z$ that $U \times B \subset W$. Then $\widetilde{F} \mid U \times B$ induces the map $\tilde{f}: U \rightarrow \Gamma(X)$ which is an extension of $f$. Hence $\Gamma(X)$ is an ANR.

Since $Y$ is complete-metrizable, $X$ is locally complete-metrizable, hence completemetrizable (cf. [BP, Chapter II, Theorem 4.1]). Since $B$ is nondiscrete, $B$ has a cluster point $b_{\infty}$. From local triviality of $p$, there is an open neighborhood $U$ of $b_{\infty}$ in $B$ and a homeomorphism $\varphi: \operatorname{cl} U \times Y \rightarrow p^{-1}(\operatorname{cl} U)$ such that $p \varphi=\pi_{\operatorname{cl} U}$. Since $Y$ has no isolated point, $Y$ admits a compatible complete metric $d_{Y}$ such that each component of $Y$ has diam $>1$ (cf. [BP, Chapter II, Theorem 3.2]). We may assume that $\left(Y, d_{Y}\right)$ has the property $(\mathrm{h})$ in Lemma 2 . Let $\rho$ be the product metric on $\operatorname{cl} U \times Y$ defined by a metric for $\operatorname{cl} U$ and $d_{Y}$. And let $d$ be a compatible complete metric for $X$ extending the metric on $p^{-1}(\mathrm{cl} U)$ induced from $\rho$ by $\varphi$ (cf. 
[BP, Chapter II, Theorem 3.2]). Then the sup-metric $\hat{d}$ on $\Gamma(X)$ is a compatible complete metric.

Let $\left(b_{i}\right)_{i \in \mathbf{N}}$ be a sequence of distinct points in $U \backslash\left\{b_{\infty}\right\}$ which converges to $b_{\infty}$. For each $i \in \mathbf{N}$, let

$$
\Gamma_{i}(X)=\left\{s \in \Gamma(X) \mid \pi_{Y} \varphi^{-1} s\left(b_{j}\right)=\pi_{Y} \varphi^{-1} s\left(b_{\infty}\right) \text { for all } j \geq i\right\} .
$$

Thus we have a tower $\Gamma_{1}(X) \subset \Gamma_{2}(X) \subset \cdots(\subset \Gamma(X))$. We will show that this tower satisfies the properties (a) and (b) relative to $\hat{d}$. Then $\Gamma(X)$ is an $l_{2}$-manifold by Toruńczyk's characterization.

(a) Let $A \subset I^{n}$ be a compactum, $f: I^{n} \rightarrow \Gamma(X)$ a map with $f(A) \subset \Gamma_{i}(X)$ and $\varepsilon>0$. Then $f$ induces the map $F: I^{n} \times B \rightarrow X$ such that $p F=\pi_{B}$ and

$$
\pi_{Y} \varphi^{-1} F\left(a, b_{k}\right)=\pi_{Y} \varphi^{-1} F\left(a, b_{\infty}\right) \quad \text { for all } a \in A \text { and } k \geq i .
$$

Let $\delta=\delta(\varepsilon)>0$ in the property (h) for $\left(Y, d_{Y}\right)$. Since $I^{n} \times B$ is compact, $\pi_{Y} \varphi^{-1} F$ is uniformly continuous. Thus we can choose $j \geq i$ so that

$$
d_{Y}\left(\pi_{Y} \varphi^{-1} F\left(z, b_{k}\right), \pi_{Y} \varphi^{-1} F\left(z, b_{\infty}\right)\right)<\delta \quad \text { for all } z \in I^{n} \text { and } k \geq j .
$$

By using (h) and the Homotopy Extension Theorem [Hu, Chapter IV, Theorem 1.2], we can obtain a map $g^{\prime}: I^{n} \times \operatorname{cl} U \rightarrow Y$ such that

$$
\begin{aligned}
& g^{\prime}\left|A \times \operatorname{cl} U \cup I^{n} \times \operatorname{bd} U=\pi_{Y} \varphi^{-1} F\right| A \times \operatorname{cl} U \cup I^{n} \times \operatorname{bd} U, \\
& g^{\prime}\left(z, b_{k}\right)=\pi_{Y} \varphi^{-1} F\left(z, b_{\infty}\right) \text { for all } z \in I^{n} \text { and } j \leq k \leq \infty \\
& g^{\prime} \text { and } \pi_{Y} \varphi^{-1} F \mid I^{n} \times \operatorname{cl} U \text { are } \varepsilon \text {-homotopic. }
\end{aligned}
$$

Define a map $G: I^{n} \times B \rightarrow X$ with $p G=\pi_{B}$ as follows:

$$
G(z, b)= \begin{cases}\varphi\left(b, g^{\prime}(z, b)\right) & \text { if } b \in \operatorname{cl} U \\ F(z, b) & \text { otherwise }\end{cases}
$$

Then $G$ induces the map $g: I^{n} \rightarrow \Gamma_{j}(X)$ such that $g|A=f| A$ and for each $z \in I^{n}$,

$$
\begin{aligned}
\hat{d}(g(z), f(z)) & =\sup \{d(g(z)(b), f(z)(b)) \mid b \in \operatorname{cl} U\} \\
& =\sup \left\{d_{Y}\left(\pi_{Y} \varphi^{-1} G(z, b), \pi_{Y} \varphi^{-1} F(z, b)\right) \mid b \in \operatorname{cl} U\right\} \\
& =\sup \left\{d_{Y}\left(g^{\prime}(z, b), \pi_{Y} \varphi^{-1} F(z, b)\right) \mid b \in \operatorname{cl} U\right\}<\varepsilon .
\end{aligned}
$$

(b) For each $\varepsilon>0$, let $\delta=\delta(\varepsilon)>0$ in (h) for $\left(Y, d_{Y}\right)$. We may assume $\delta<1$. Let $f: I^{n} \rightarrow \Gamma_{i}(X)$ be a map. Then $f$ induces the map $F: I^{n} \times B \rightarrow X$ such that $p F=\pi_{B}$ and

$$
\pi_{Y} \varphi^{-1} F\left(z, b_{k}\right)=\pi_{Y} \varphi^{-1} F\left(z, b_{\infty}\right) \quad \text { for all } z \in I^{n} \text { and } k \geq i .
$$

From compactness, there exist $y_{1}, \cdots, y_{m} \in Y$ such that

$$
\pi_{Y} \varphi^{-1} F\left(I^{n} \times B\right) \asymp B\left(y_{1}, \delta / 3\right) \cup \cdots \cup B\left(y_{m}, \delta / 3\right),
$$

where $B(y, r)=\left\{x \in Y \mid d_{Y}(x, y)<r\right\}$. For each $j=1, \ldots, m$, choose a point $z_{j} \in B\left(y_{j}, \delta\right) \backslash B\left(y_{j}, 2 \delta / 3\right)$. (Since each component of $Y$ had diam $>1$, we can choose such a point.) Using the Homotopy Extension Theorem and (h), we have a map $g_{j}: Y \rightarrow Y$ such that $g_{j}\left(B\left(y_{j}, \delta / 3\right)\right)=z_{j}, g_{j} \mid Y \backslash B\left(y_{j}, \delta\right)=$ id and $g_{j}$ is $\varepsilon$-homotopic to id. Then it follows

(*) $\max \left\{d_{Y}\left(y, g_{j}(y)\right) \mid j=1, \ldots, m\right\}>\delta / 3$ for each $y \in \pi_{Y} \varphi^{-1} F\left(I^{n} \times \operatorname{cl} U\right)$. 
Again using the Homotopy Extension Theorem, we can obtain a map $g^{\prime}: I^{n} \times \operatorname{cl} U \rightarrow$ $Y$ such that

$$
\begin{aligned}
& g^{\prime}\left|I^{n} \times \mathrm{bd} U=\pi_{Y} \varphi^{-1} F\right| I^{n} \times \mathrm{bd} U, \\
& g^{\prime}\left(z, b_{k}\right)=\pi_{Y} \varphi^{-1} F\left(z, b_{\infty}\right) \quad \text { if } i+m<k \leq \infty, \\
& g^{\prime}\left(z, b_{i+j}\right)=g_{j} \pi_{Y} \varphi^{-1} F\left(z, b_{i+j}\right) \quad \text { if } j=1, \ldots, m, \\
& g^{\prime} \text { is } \varepsilon \text {-homotopic to } \pi_{Y} \varphi^{-1} F \mid I^{n} \times \operatorname{cl} U \text { rel. } I^{n} \times \operatorname{bd} U .
\end{aligned}
$$

As in the proof of (a), define a map $G: I^{n} \times B \rightarrow X$ with $p G=\pi_{B}$ by using the above $g^{\prime}$. It is easy to see that $G$ is f.p. $\varepsilon$-homotopic to $F$. Then $G$ induces the map $g: I^{n} \rightarrow \Gamma_{i+m+1}(X)$ which is $\varepsilon$-homotopic to $f$. We show $\operatorname{dist}\left(f\left(I^{n}\right), g\left(I^{n}\right)\right) \geq \delta / 6$. Suppose that $\hat{d}\left(f(z), g\left(z^{\prime}\right)\right)<\delta / 6$ for some $z, z^{\prime} \in I^{n}$. Then

$$
\begin{gathered}
d_{Y}\left(\pi_{Y} \varphi^{-1} F\left(z, b_{\infty}\right), \pi_{Y} \varphi^{-1} F\left(z^{\prime}, b_{\infty}\right)\right)=d_{Y}\left(\pi_{Y} \varphi^{-1} F\left(z, b_{\infty}\right), g^{\prime}\left(z^{\prime}, b_{\infty}\right)\right) \\
=d\left(F\left(z, b_{\infty}\right), G\left(z^{\prime}, b_{\infty}\right)\right) \leq \hat{d}\left(f(z), g\left(z^{\prime}\right)\right)<\delta / 6 .
\end{gathered}
$$

And for each $j=1, \ldots, m$,

$$
\begin{aligned}
d_{Y}( & \left.\pi_{Y} \varphi^{-1} F\left(z, b_{\infty}\right), g_{j} \pi_{Y} \varphi^{-1} F\left(z^{\prime}, b_{\infty}\right)\right) \\
& =d_{Y}\left(\pi_{Y} \varphi^{-1} F\left(z, b_{i+j}\right), g_{j} \pi_{Y} \varphi^{-1} F\left(z^{\prime}, b_{i+j}\right)\right) \\
& =d_{Y}\left(\pi_{Y} \varphi^{-1} F\left(z, b_{i+j}\right), g^{\prime}\left(z^{\prime}, b_{i+j}\right)\right)=d\left(F\left(z, b_{i+j}\right), G\left(z^{\prime}, b_{i+j}\right)\right) \\
& \leq \hat{d}\left(f(z), g\left(z^{\prime}\right)\right)<\delta / 6 .
\end{aligned}
$$

Hence for each $j=1, \ldots, m$,

$$
d_{Y}\left(\pi_{Y} \varphi^{-1} F\left(z^{\prime}, b_{\infty}\right), g_{j} \pi_{Y} \varphi^{-1} F\left(z^{\prime}, b_{\infty}\right)\right)<\delta / 3 .
$$

This contradicts $(*)$. Therefore $d\left(f(z), g\left(z^{\prime}\right)\right) \geq \delta / 6$ for any $z, z^{\prime} \in I^{n}$, that is, $\operatorname{dist}\left(f\left(I^{n}\right), g\left(I^{n}\right)\right) \geq \delta / 6$. The proof is completed.

3. The space of fiber-preserving maps. Let $p: X \rightarrow B$ and $q: Z \rightarrow B$ be given maps. By $C_{B}(Z, X)$, we denote the space of f.p. maps from $Z$ to $X$ with compact-open topology. The following is a generalization of Main Theorem which can be proved directly by the same method but it follows from Main Theorem as a corollary.

COROLlaRY. Let $q: Z \rightarrow B$ be a map of a nondiscrete compactum $Z, Y a$ complete-metrizable ANR with no isolated point and $p: X \rightarrow B$ a locally trivial bundle with fiber $Y$. Then $C_{B}(Z, X)$ is an $l_{2}$-manifold if $C_{B}(Z, X) \neq \varnothing$.

ProOF. Consider the fiber-product $X \times_{B} Z=\{(x, z) \in X \times Z \mid p(x)=q(z)\}$ of $X$ and $Z$ over $B$. Let $\pi_{X}: X \times Z \rightarrow X$ and $\pi_{Z}: X \times Z \rightarrow Z$ denote the projections. Then $\pi_{Z} \mid X \times_{B} Z$ is a locally trivial bundle with fiber $Y$ because so is $p: X \rightarrow B$. Let $\Gamma\left(X \times_{B} Z\right)$ be the space of cross-sections of this bundle. Define a $\operatorname{map} \theta: \Gamma\left(X \times_{B} Z\right) \rightarrow C_{B}(Z, X)$ by $\theta(s)=\pi_{X} \circ s$. Then it is easy to see that $\theta$ is a homeomorphism. The result follows from Main Theorem.

As mentioned in Lemma 3, any locally trivial bundle with ANR fiber is an ANFR. Besides a Hurewicz fibration between ANR's and a proper strongly regular map onto a finite-dimensional space with ANR fibers are also ANFR's (cf. [Ya, 1.2]). We have the f.p. Homotopy Extension Theorem [Ya, 1.1 (iv)] and the f.p. version of Lemma 2. By the same method as Main Theorem, we can prove the following theorem which is analogous to the above corollary but in a little different setting. 
THEOREM. Let $q: Z \rightarrow B$ be a map of a compactum and $p: X \rightarrow B$ an ANFR such that $X$ is complete-metrizable. Then $C_{B}(Z, X)$ is an $l_{2}$-manifold if $C_{B}(Z, X) \neq \varnothing, q^{-1}(b)$ is nondiscrete and $p^{-1}(b)$ has no isolated point for some $b \in B$.

Because of similarity, the proof is left to the reader. (We can also prove the relative version of this theorem, i.e., the f.p. version of [To $\mathbf{T}_{\mathbf{1}}$, Theorem 5.5].) Here we give the proof of the f.p. version of Lemma 2 .

LEMma $2^{\prime}$. Let $p: X \rightarrow B$ be an ANFR and $d_{0}$ a metric for $X$. Then $X$ has a compatible metric $d \geq d_{0}$ with the following property

$\left(\mathrm{h}^{\prime}\right)$ For each $\varepsilon>0$, there is a $\delta=\delta(\varepsilon)>0$ such that any two $\delta$-near f.p. maps of an arbitrary space $Z$ with a map $q: Z \rightarrow B$ to $X=(X, d)$ are f.p. $\varepsilon$-homotopic.

ProOF. Since $X=\left(X, d_{0}\right)$ can be isometrically embedded in a normed linear space $E$ as a closed set (cf. [BP, Chapter II, Corollary, 1.1]), we have an f.p. closed embedding $i: X \rightarrow B \times E$ such that $\pi_{E} i$ is an isometry, where $\pi_{E}: B \times E \rightarrow E$ is the projection. Identify $X$ with $i(X) \subset B \times E$ and $p=\pi_{E} \mid$. Let $d_{1}$ be the product metric on $B \times E$. Then $d_{1} \geq d_{0}$ on $X$. Since $p: X \rightarrow B$ is an ANFR, there is an f.p. retraction $r: G \rightarrow X$ of a neighborhood $G$ of $X$ in $B \times E$. For a subset $S \subset B \times E$, we denote

$$
\operatorname{conv}_{B} S=\bigcup\left\{\{b\} \times \operatorname{conv} \pi_{E}(S \cap\{b\} \times E) \mid b \in B\right\}
$$

where conv $A$ denotes the convex hull of $A \subset E$. For each $x \in X$ and each neighborhood $V$ of $x$ in $X$, there is a basic open set $U=U_{1} \times U_{2}$ in $B \times E$ such that $\pi_{E}(U)=U_{2}$ is convex and $x \in U \subset r^{-1}(V)$. Let $W=U \cap X$. Then $\operatorname{conv}_{B} W \subset U \subset G$ and $r\left(\operatorname{conv}_{B} W\right) \subset r(U) \subset V$. By the same way as [Mi, Lemma 2.1], we have a compatible metric $d \geq d_{1}$ on $X$ with the following property:

(c) To every $\varepsilon>0$ corresponds a $\delta=\delta(\varepsilon)>0$ such that if $S \subset X$ with $\operatorname{diam} S<\delta$ then $\operatorname{conv}_{B} S \subset G$ and $\operatorname{diam} r\left(\operatorname{conv}_{B} S\right)<\varepsilon$.

By standard arguments, $\left(h^{\prime}\right)$ follows from (c). (Cf. [Hu, Chapter IV, Theorem 1.1].)

\section{REFERENCES}

[BP] C. Bassaga and A. Pełczyński, Selected topics in infinite-dimensional topology, Monogr. Mat. 58, PWN, Warsaw, 1975.

[DT] T. Dobrowolski and H. Toruńczyk, Separable complete ANR's admitting a group structure are Hilbert manifolds, Topology Appl. 12 (1981), 229-235.

[Du] J. Dugundji, Topology, Allyn \& Bacon, Boston, Mass., 1966.

[E] J. Eells, Jr., On geometry of function spaces, Symp. Intern. de Topologia Algebraica Univ. Nacional Autónoma de México and UNESCO, Mexico City, 1958, pp. 303-308.

[Ge] R. Geoghegan, On spaces of homeomorphisms, embeddings and functions, I, Topology 11 (1972), 159-177.

[Hu] S.-T. Hu, Theory of retracts, Wayne State Univ. Press, 1965.

[Mi] E. Michael, Uniform AR's and ANR's, Compositio Math. 39 (1979), 129-139.

[TO1] H. Torunczyk, Concerning locally homotopy negligible sets and characterization of $l_{2}$-manifolds, Fund. Math. 101 (1978), 93-110.

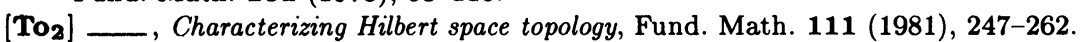

[Tos] - A correction of two papers concerning Hilbert manifolds, Fund. Math. 125 (1985), 89-93.

[Ya] T. Yagasaki, Fiber shape theory, Tsukuba J. Math. 9 (1985), 261-277. JAPAN

INSTitute of Mathematics, University of TSUKUba, TSUKUba, Ibaraki, 305 